\title{
Chemistry of materials science phenomena in high-intensity discharge light sources*
}

\author{
Z. Tóth ${ }^{\ddagger}$ and H. Lovas \\ General Electric Consumer \& Industrial, 1340 Budapest Váci út 73-77, Hungary
}

\begin{abstract}
Continuous development of light sources is required from energy-saving considerations (enhancing luminous efficacy) and an environmental protection point of view (lamps that are easy to recycle or made of environmentally friendly materials). This paper summarizes some of the recent results of materials science research with special attention to chemical aspects. The results will be cited from the field of discharge chemistry, electrodes, ceramics, and other materials.
\end{abstract}

Keywords: HID lamps; polycrystalline alumina; discharge tubes; sealing; discharge chemistry; electrodes.

\section{INTRODUCTION}

Lighting today is considered a simple service in our everyday life. The increasing cost of energy and maintenance, the need for environmentally friendly systems, the demand for reduction of energy usage, and hence the emission of greenhouse gases, makes the continuous development of existing and future light sources necessary. Nontypical applications such as medical or horticultural lighting are another area where very special development activity is necessary. During these developments, the light source manufacturers have to leverage up-to-date materials and rely on widespread knowledge of the physical and chemical phenomena that take place during the operation of the lamp. Manufacturing of these products also requires new technologies that are based on dedicated research of physical processes, chemical reactions, and materials science of the substances used in the light source.

High-intensity discharge (HID) lamps play a major role in today's lighting. The two major families: the high-pressure sodium (HPS) lamp and the ceramic metal halide (CMH) lamp provide a wide range of luminous efficacy (up to 150 lumen/W, HPS) or excellent color-rendering index ( $\mathrm{Ra}=90+$, $\mathrm{CMH}$ ). The former offers very efficient lighting with acceptable color, hence they are used mainly in public, road, or industrial lighting. The latter offers excellent color, and application areas are decorative lighting of buildings, shops, and offices, both in exterior as well as interior applications. Examples of the two families can be seen in Fig. 1.

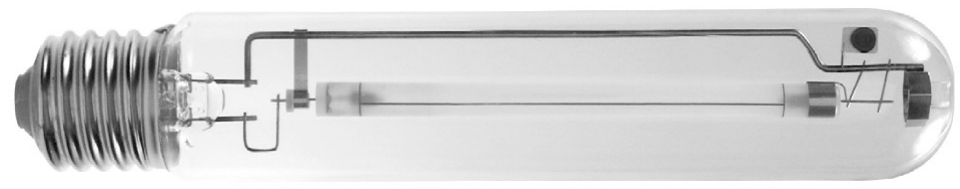

Fig. 1a Lucalox XO HPS lamp.

\footnotetext{
*Paper based on a presentation at the $12^{\text {th }}$ International IUPAC Conference on High Temperature Materials Chemistry (HTMC-XII), 18-22 September 2006, Vienna, Austria. Other presentations are published in this issue, pp. 1635-1778.

¥Corresponding author
} 

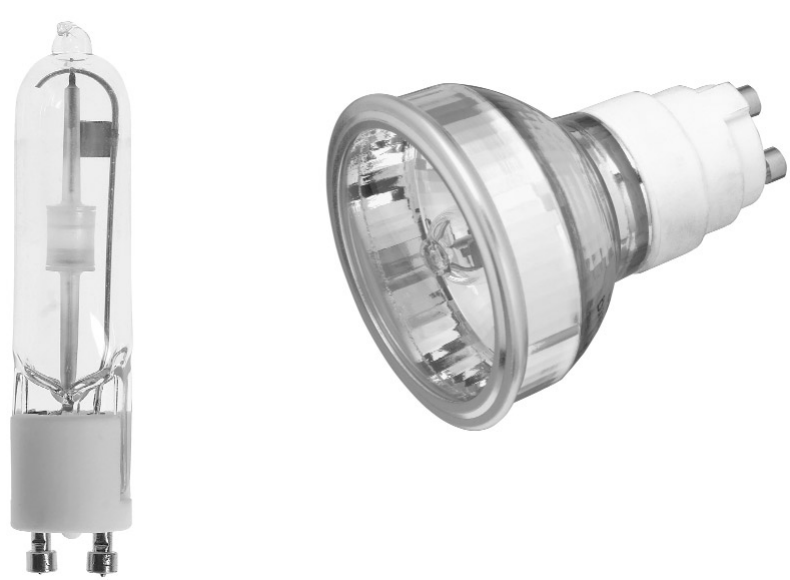

Fig. 1b CMH 20-W Supermini lamp in single-ended and precise MR16 finish.

In HID lamps, the photons used for lighting are emitted from a discharge plasma generated between two electrodes. Figure 2 shows the schematics of HID lamps. The major parts-the electrodes, the elements of the discharge, the ceramic arc tube, and the sealing in-between the arc tube and electric feedthrough - are shown. Although grouping of the research activities is not easy or almost impossible due to the interactions between the phenomena of the different areas, this paper describes and takes examples from the following key science and technology areas of the HID light sources: plasma physics and discharge chemistry, electrodes, ceramics. Other important areas of the lighting will not be covered.

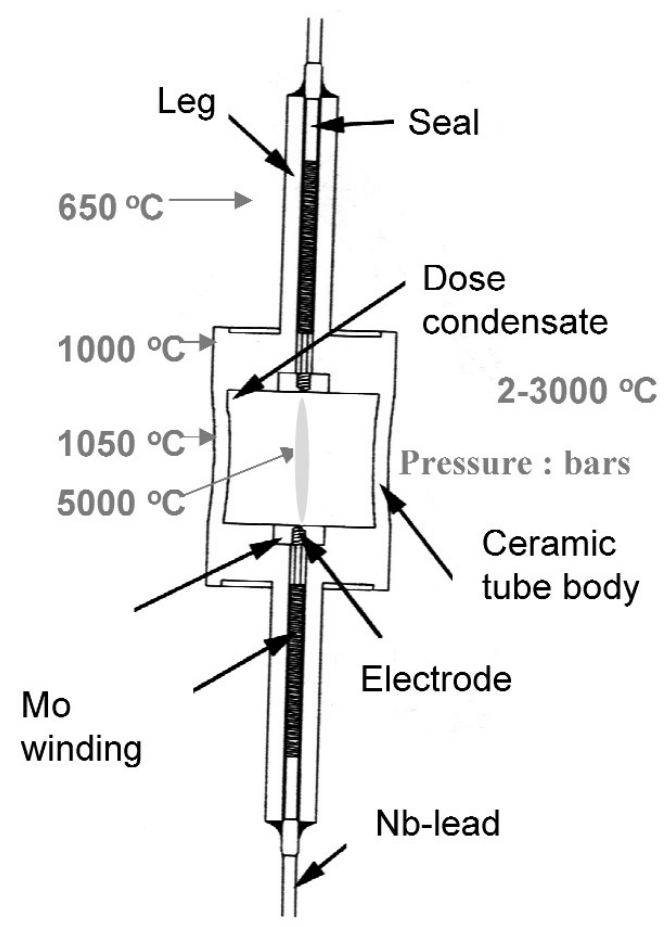

Fig. 2 Construction and temperatures of the major components of a $\mathrm{CMH}$ arc tube. 


\section{DISCHARGE CHEMISTRY}

The photons in the HID lamps are generated in a discharge. HPS lamps are usually dosed with $\mathrm{Na}-\mathrm{Hg}$ amalgam + Xe starting gas, the spectrum is dominated by the self-reversed Na D-lines [1]. To improve the color-rendering of the lamp, other elements must be added to the spectrum. Since the vapor pressures of the metals, which could be considered from a spectral point of view, are typically low at a temperature the lamp constituents could withstand, these metals are added in the form of metal halides, giving the family name of metal halide lamps [2]. This way, atomic and diatomic lines, molecular and continuum radiation can be observed in the lamp spectrum. The aim of the designer is to select a combination of metal halide doses which best fit the desired application from a lamp color point of view, give the best energy usage to achieve this color and illuminance level, and maintain both the color as well as the light level over as long as possible life of the product. Unfortunately, this means that a number of compromises must be made to satisfy these requirements simultaneously. Some typical dose combinations are

$$
\{\mathrm{Hg}+\mathrm{Na}+\mathrm{Sc}+\mathrm{I}\},\{\mathrm{Hg}+\mathrm{Na}+\mathrm{In}+\mathrm{Sn}+\mathrm{Tl}+\mathrm{Br}+\mathrm{I}\}, \text { or }\{\mathrm{Hg}+\mathrm{Na}+\text { lanthanides }+\mathrm{I}+\mathrm{Ca}\}
$$

In parallel with the dose optimization, arc tube geometry should be optimized as well, or even the whole system including the ballasts and fixture can be developed as a system.

Different plasma models help the designer's work, although, especially in the case of the complex metal halide systems, there are no available ab initio simulation tools that predict the spectrum of the lamp dosed with a certain salt mixture. Experimental methods with statistical evaluation, like simplex optimization or design of experiments are described as the most appropriate methods by Brumleve [3]. Determination of the thermochemical data, however, is indispensable to develop reliable plasma models, as described by Mucklejohn [4]. Input parameters for the plasma models are viscosity, electrical conductivity, thermal conductivity, and heat capacity. The calculation of these parameters requires the transport coefficients, which ultimately depends on the chemical composition. The calculation is based on the minimization of the Gibbs energy of the system. For each species of the system determination of the key thermochemical parameters like enthalpy of formation, molar enthropy, transition temperatures, enthalpies of transition, melting temperature, etc. are needed. These data are not needed only for the species dosed into the lamps, since homo- and heterocomplexes are formed in the discharge. Lamp models do require the knowledge of the composition of these gaseous or condensed species as well as their fragments and their thermochemical data like dissociation or vaporization. Hilpert et al. used the Knudsen effusion/mass spectrometry method to determine the gas-phase compositions, their partial pressures in a wide range of pressure and temperature, the computation of enthalpy and enthropy changes of the dissociation reaction of complexes, as well as equilibrium constants [5]. This method also allows determination of the factor of the enhancement of the concentration of a certain element in the discharge column. Dosing NaI-TmI ${ }_{3}$ into an alumina Knudsen cell beside the original dose, Markus et al. detected complexes resulting from the reactions between the dose, compounds like $\mathrm{Na}_{2} \mathrm{I}_{2}$ or $\mathrm{Na}_{2} \mathrm{TmI}_{5}$, also reaction products with the cell like $\mathrm{AlI}_{3}$ or $\mathrm{NaAlI}_{4}[6]$. This highlights the importance of compatibility of the dose materials with parts of the lamp. Corrosion reactions take place between the dose elements and the arc tube material, the electrodes, or the sealing (see below). Details of the methods, the calculation, and the results are described in [7] in this volume. The authors point out the importance of heterocomplex formation to the enhancement of concentration of the sodium in the plasma, leading to change of the color properties of the lamp.

Spectroscopic characterization of the lamps is also needed for better modeling. Absorption and emission spectra of the metal halides, the complexes formed, or their fragments should be also an important area of the lamp development. Spectroscopic methods are described, for instance, in [8].

Although production of up-to-date HID lamps is being made in dry boxes, where the levels of oxygen, water, and hydrogen are minimized and are kept typically in the ppm level, the effect of these impurities must also be considered during modeling of the lamp. 


\section{ELECTRODES}

Electrodes play an important role both during the starting as well as the steady-state operation of the lamp. Their primary role is to supply electrons to the discharge (in the cathode phase). Meanwhile, the electron yield is increasing by the temperature of the electrode tip (described by the Richardson-Dushman equation), the temperature should be kept in control to reduce the evaporation of the electrode (or ultimately its melting). Because of the high melting point and low evaporation properties, tungsten is the most widely used electrode material in HID lamps. Detailed description of the electrodes can be found in [1] or [2]. For optimum efficacy, the electrode loss should also be kept as low as possible. Hence, beside the actual geometry design of the electrode, decreasing its work function is a key element for improving energy balance of the lamp. Work functions of the electrodes can be decreased by the adsorption of a monolayer of activator atoms on the surface of the electrodes. Meanwhile, during the operation of the lamp, the elements of the discharge can form this layer; during start-up, the emitter material stored on the electrode can have a beneficial effect for easy start-up, hence reducing the sputtering effect in the transient period. In the HPS lamps, Ba-containing materials are used as emission materials.

Change of the work function during the operation of the lamp can change the electrical properties of the lamp. Beck et al. have monitored the work function of HPS lamps as a function of the sintering condition of the emission material and as a function of the aging of the lamp [9]. Beside the conventional Kelvin probe measurement, they have developed the so-called work function spectroscopy (WFS), which is capable of determining the work function with very good spatial resolution. WFS is based on the fact that if a conducting material surface is irradiated by electrons (in an Auger electron spectroscopy, AES, chamber in this case) the edge of the secondary electron spectrum depends on the work function of the material through which the secondary electrons had to pass while they were liberated from the surface. Since the work was done in an AES, combining the WFS with the elemental analysis capability of the AES, elemental composition-work function mapping of the surface can be undertaken. The measurements were made with no plasma exposure, so the effect of dose constituent layers was separated from the change of the electrode material. For the electrodes without aging, they have found an increasing work function with increasing temperature, which they concluded from the changing of the composition of the $\mathrm{Ba}, \mathrm{C}$, and $\mathrm{O}$ on the surface. Meanwhile, they note also that the measurements were made at room temperature, but at the operating temperature due to the desorption, the effect of surface contaminants is less. Comparing the lamps at their initial state to that after $2000 \mathrm{~h}$ aging, they concluded the increase of the work function with aging time as well accompanied by disappearance of the Ba signal from the surface. This indicates that the thickness of emission material coverage on the electrode is decreasing. The hot end electrode, however, has not shown significant change of the work function, indicating that the work started can be continued to give further explanations.

Due to the chemical reaction between halides and emitter materials, meta halide lamps use electrodes without e-mix. Fortunately, even such a complex system of electrode + different dose systems can be modeled in good agreement with the measured data. Benilov and coworkers have described such a model of current transfer to cathodes in metal halide plasmas [10]. They concluded that, due to the non-monotonous dependence of the work function of the surface coverage of electrode by the alkali atoms, the dependence of the work function on the cathode surface temperature is also non-monotonic. They also concluded that even $\sim 1 \%$ of alkali vapor in the plasma can have significant effect on the work function. Addition of metal halides to the gas phase decreases the temperature of the electrode as well as having an effect on the stability range of the different operating modes of the electrodes. The work function reduction effect was stronger for $\mathrm{Cs}$, than $\mathrm{Na}$, and differences between the stability range of different modes and current-voltage characteristics have been found. Luijks et al. have derived the effective work function of the electrodes by temperature mapping of the electrode in a transparent YAG system accompanied by modeling calculations [11]. They have measured the effect of adding a Dy-containing salt to the discharge. They have found that meanwhile the addition of NaTl-iodide did not prac- 
tically change the effective work function of the electrode compared to the result measured in pure $\mathrm{Hg}$ discharge. The addition of Dy-containing salt decreased it by $\sim 1 \mathrm{eV}$ (they called this the gas-phase emitter effect). They explained their result by the increased tendency of Dy to absorb on the W surface compared to $\mathrm{Na}$ or $\mathrm{Tl}$, due to the higher enthalpy of absorption of Dy $(-529 \mathrm{~kJ} / \mathrm{mol})$ vs. $\mathrm{Na}$ or $\mathrm{Tl}$ $(-225 \mathrm{~kJ} / \mathrm{mol}$ and $-315 \mathrm{~kJ} / \mathrm{mol}$, respectively). In agreement with the previous authors, they have also observed the reduction of cathode temperature as well as accompanied by change of the operating mode of the electrodes.

Another way to improve the performance of the electrode is doping it with Th. Thoriated electrodes result in lower evaporation rates than for pure $\mathrm{W}$ electrodes and have a positive role in the regeneration cycles as well [2]. Due to environmental protection considerations, the replacement of the radioactive Th-doped electrodes is desirable. To achieve this, phenomena related to the Th-doped operation must be fully understood. Lamouri et al. compared the performance lumen maintenance and electrical parameters stability of thoriated electrodes vs. pure W ones in identical lamp design [12]. They found that operating the lamps on rapid cycling, which emphasizes the starting-related phenomena (i.e., lamps were switched off after only 2 min of operation and were switched on again after 30 min of cooling-down period), the pure $\mathrm{W}$ electrodes show higher lumen depreciation in the lower fill pressure case (up to 75 mbar). Meanwhile, increasing the arc tube fill pressure above 100 mbar resulted in no significant difference. The authors explain this by the higher sputtering rate at lower fill pressure, meanwhile, the lumen maintenance drop found has been explained by thoria depletion of the thoriated electrodes. One has to note that lamps made with both electrode types contained $\mathrm{ThI}_{4}$ as a dose constituent. There was a significant difference in the loss rate of the $\mathrm{ScI}_{3}$ dose due to the increased rate of reaction of the $\mathrm{ScI}_{3}$ dose with the $\mathrm{ThO}_{2}$ in the electrodes. The effect of this was better lumen maintenance and less operating voltage rise for pure $\mathrm{W}$ electrode, than in the case of the thoriated one. Boroczki et al. have used AES to follow the enrichment of the Th adatoms on the electrode surface [13]. Beside the classical Langmuir-type segregation behavior of the Th adatoms to the surface, they have described a new type of Th enrichment on the free surface of the electrode. This kind of adatom coverage appeared only if the current density of the bombarding electrodes was sufficiently high. They also detected the dissociation of thoria by measuring the Th and $\mathrm{O}$ concentration on the surface. They believe the knowledge of the $\mathrm{O}$ coverage may be used to measure quantitatively the $\mathrm{O}$ activity that allows the dissociation. Since during the anodic phase of the operation of the lamps the electrode is bombarded by electrons, this finding has an important role in the starting phenomenon of the metal halide lamps. Similarly to Beck et al., the authors also point out the importance of the electrode-plasma interactions in real operating lamps.

Attention must be paid to the interaction between the ballast system and electrode performance. Lamouri et al. compared the lamp performance operating on $50 \mathrm{~Hz}$ electromagnetic ballast vs. a high frequency (typically, a few $\mathrm{kHz}$ ) electronic ballast. They concluded that for the same average current, the peak temperature of electrode is lower for the high-frequency ballast by $\sim 200 \mathrm{~K}$. This equates in general terms to $\sim 10-20 \%$ better performance on the type of electronic ballast used by the authors.

Summarizing the work related to the electrode systems, one can conclude that work function determination is still among the key activities. Describing its change during the operation and leveraging in designing the optimal dose system is taking place. Comparing results provided by direct, plasma-free measurements to the results of ones derived via temperature or electrical parameters accompanied by modeling can also give useful information. Understanding thoriated systems and the chemical reactions with the dose components will also provide better electrode designs for application areas beside lighting. Understanding starting processes, reducing sputtering phenomena can contribute to better lumen maintenance and better reliability of the lamps as well. These activities require knowledge of different thermochemical parameters like enthalpy of adsorption, similarly as described above. Ultra-high-purity electrodes for miniaturized lamps (SuperMINI or Short arc) create even higher demands for the electrodes. Fortunately, very good electrode models help the work of the lamp designers [10]. 


\section{CERAMICS AND SEALINGS}

The first commercial application of the arc tube material made of ceramics was the HPS lamp from the beginning of the 1960s. The two key requirements at that time were the translucency and vacuum tight enclosure of the discharge tube. Ceramic technology has advantages in the metal halide family as well: the higher wall temperature enables higher vapor pressure of the discharge elements, resulting in better performance of the lamp. The time history of the resulting CMH lamp family is described in [14]. The status quo of the CMH lamps has been recently summarized by Kappen [15].

Recent applications, such as automotive discharge lamps and projector lamps, require point-like, easy-to-focus light sources. To achieve this, one has to move from the translucent ceramic arc tube to the transparent one. The problem with this change can be found in Wei's recent article [16]. Kappen and Wei describe two major routes for making transparent ceramic materials: one is based on the current alumina (single-crystalline sapphire or submicron grain-sized ceramic tube) or alternative materials (YAG, $\mathrm{AlON}, \mathrm{MgAl}_{2} \mathrm{O}_{4}, \mathrm{Y}_{2} \mathrm{O}_{3}$ ).

Both alumina as well as alternative materials have to fulfil the following requirements beside transparency: stability (properties with AlON, e.g., see [17], chemical compatibility with the discharge dose elements, mechanical compatibility with the metallic current feedthrough in the sealing, and, last but not least, easy to manufacture either as a straight tube or in any other more advanced arc tube geometry.

Chemical compatibility means no loss of the dose as well as no corrosive reaction between the dose elements and the arc tube material. Loss of the $\mathrm{Na}$, for instance, can happen via diffusion via the ceramic tube material. The transport through the polycrystalline ceramic arc tube consists of several steps. This complex process was separated into different parts, and the two most important partial processes (Na diffusion through the polycrystalline alumina wall, the solid-state reaction between alumina and $\mathrm{Na}$ ) were examined separately from the disturbing effect by Lovas et al. [18]. They investigated the outer surface of the ceramic arc tubes of experimental lamps burned for different times by X-ray photoelectron spectroscopy (XPS) in order to examine the different chemical and physicochemical processes in the wall of the ceramic arc tube during the lifetime of the lamp. The considerable amount of $\mathrm{Na}$ detected on the outer surface of the ceramic discharge tube suggested that Na passes through the ceramic wall and accumulates on the outer surface of the tube. As the source of the $\mathrm{Na}$ is inside the arc tube, one would expect increasing $\mathrm{Na}$ level coming from the outer surface toward the arc tube. On the contrary, Na accumulation could be detected on the outer surface of the arc tube. The separation of $\mathrm{Na}$ from the surface to the gas phase can be blocked by chemical bonding formed between $\mathrm{Na}$ and the alumina. This can cause Na-layer pile-up on the outer surface of the alumina tube. It was concluded from these investigations that Na leaves the ceramic tube mainly by the way of diffusion, because the analytical examinations showed higher $\mathrm{Na}$ concentration in the middle area of the ceramic tube then at the edge. From the diffusion profile of model experiments (alumina discs subjected to $\mathrm{Na}$ vapor in a reactor chamber) they concluded that the type-B diffusion model describes best the diffusion process: grain boundary diffusion plays an important role in the formation of the Na diffusion profile, but volume diffusion takes place also. One has to note that the theory was not validated at higher temperature. At higher temperature, other processes (formation of some $\mathrm{Na}-\mathrm{O}-\mathrm{Al}$ compounds) could have an influence on the diffusion mechanism.

In [18], the authors examined and modeled the possibility of the solid-state reaction between $\mathrm{Na}$ and the polycrystalline alumina arc tube under lamp conditions as well. The investigation indicated the following phases: $\mathrm{NaAlO}_{2}$ after shorter heat treatment, the formation of some another $\mathrm{Na}-\mathrm{O}-\mathrm{Al}$ compounds after longer heat treatment, such as $\mathrm{NaAl}_{11} \mathrm{O}_{17}(\mathrm{Na}-\beta$-alumina) with hexagonal structure, which is poorer in $\mathrm{Na}$. This suggests that $\mathrm{NaAlO}_{2}$ with orthorhombic structure formed can be the stable product of these solid-state reactions. Using another alumina powder with more disordered structure, the solid-state reaction resulted in another $\mathrm{Na}-\mathrm{O}-\mathrm{Al}$ compound: $\mathrm{NaAl}_{23} \mathrm{O}_{35}$ with hexagonal structure. The actual structure of the alumina seems to affect the conversion of the reaction. 
Even more severe reactions can happen in the case of the CMH lamps. Markus et al. describe different transport reactions driven by the high-temperature gradient inside the arc tube. The reaction

$$
\mathrm{AlI}_{3}(\mathrm{~g})+\mathrm{Al}_{2} \mathrm{O}_{3}(\mathrm{~s})=3 \mathrm{AlOI}(\mathrm{g})
$$

can transport the arc tube material from the hot part and deposit in the cold part [6]. The source of the $\mathrm{AlI}_{3}$ can be, for instance, the reaction

$$
\mathrm{DyI}_{3}(\mathrm{~g})+\mathrm{Al}_{2} \mathrm{O}_{3}(\mathrm{~s})=\mathrm{DyAlO}_{3}(\mathrm{~s})+\mathrm{AlI}_{3}(\mathrm{~g})
$$

Solution-precipitation transport mechanism in the molten dose driven by temperature gradient in the lamp has also been described. The corrosive reactions in CMH lamps are described in detail in this volume as well [7]. Wei points out that similar reactions can take place between the dose material and alternative arc tube material as well [16].

Beside the corrosive reactions of the arc tube, the life-limiting factor can be leakage of the seals in the arc tube, that is, the connection between the ceramic arc tube material and the metallic current feedthrough. The primary reason for the leakage is believed to be thermomechanical stresses induced by the different heat expansion properties of the phases formed during the lamp-making process or relaxation of the compressive stresses formed initially. Seal structure and connecting mechanical properties are described in [19] and [20] for HPS and CMH lamps, respectively. Interface layer formation between the sealing frit material (which is a mixture of different oxides) and the ceramic arc tube is obvious, meanwhile, chemical reaction has also been reported between the metal feedthrough and the frit material [21]. Corrosive reactions have been described between Mo and halide mixtures in a heattreated quartz tube to model the quartz metal halide system as well [22].

As a future solution, sealing made of composite materials composed of metal and ceramic constituents (so called CERMET) has been reported in patent applications, see, for example [23].

\section{SUMMARY}

Materials science is a key research and development area for light-source development. Understanding the chemical reactions occurring in the high-temperature zones of the lamp is vital both from lamp efficacy enhancement as well as a reliability improvement point of view. Basic research and fundamental data are needed for better thermochemical and mechanical modeling and understanding lumen maintenance and reliability. These findings emphasize again that lamps must be considered as a system, and discharge properties must be developed together with the material parts (arc tube material and matching sealing material) of this system.

\section{ACKNOWLEDGMENTS}

The authors thank Stuart Mucklejohn for the fruitful discussions and the careful reading of the manuscript.

\section{REFERENCES}

1. J. J. de Groot, J. A. J. M. van Vliet. The High Pressure Sodium Lamp, p. 252, Kluwer Technische Boeken B.V.-Deventer-Antwerpen (1986).

2. J. F. Waymouth. Electric Discharge Lamps, M.I.T. Press, Cambridge, MA (1971).

3. T. Brumleve. HID Technology Workshop Report, 11/2005, Washington D.C.

4. S. A. Mucklejohn. COST-529 Materials Workshop Meeting, 2/2006, Budapest.

5. K. Hilpert, U. Niemann. Thermochim. Acta 299, 49 (1997).

6. T. Markus, U. Niemann, K. Hilpert. J. Phys. Chem. Solids 66, 372 (2005). 
7. T. Markus, K. Hilpert. VII International Conference on Molten Slags Fluxes and Salts, The South African Institute of Mining and Metallurgy (2004).

8. Zhu. Ph.D. thesis, TU Eindhoven (2005).

9. I. Beck, V. K. Josepovits, J. Sneider, Z. Toth. J. Phys. D: Appl. Phys. 38, 3865 (2005).

10. M. S. Benilov, M. D. Cunha, G. V. Naidis. J. Phys. D: Appl. Phys. 38, 3155 (2005).

11. G. M. J. F. Luijks, S. Nijdam, H. v. Esveld. J. Phys. D.: Appl. Phys. 38, 3163 (2005).

12. A. Lamouri, A. Naruka, J. Sulcs, C. V. Varanasi, T. R. Brumleve. J. Phys. D.: Appl. Phys. 38, 3028 (2005).

13. Á. Böröczki, I. Gaál, S. Gurbán, M. Menyhárd, E. Horváth, A. L. Tóth, L. Petrás, L. Balázs. Int. J. Refractory Metals Hard Mater. 24, 343 (2005).

14. S. A. Mucklejohn. Proceedings LS-8 International Symposium on the Science and Technology of Light Sources, Greifswald, Germany (1998).

15. T. G. M. M. Kappen. J. Phys. D: Appl. Phys. 38, 3033 (2005).

16. G. C. Wei. J. Phys. D: Appl. Phys. 38, 3057 (2005).

17. H. X. Willems, M. M. R. M. Hendrix, R. Metselaar, G. de With. J. Eur. Ceram. Soc. 10, 327 (1992).

18. H. Lovas, V. K. Josepovits, Z. Tóth, M. Tóth. Mater. Sci. Forum 537-538, 405 (2007).

19. Z. Tóth, Z. Koltai, G. Steinbach, A. Juhász, K. Váradi. J. Phys. D: Appl. Phys. 38, 3047 (2005).

20. Z. Koltai, K. Váradi, J. Dakin. Proceedings of Fourth Conference on Mechanical Engineering, Budapest 367 (2004).

21. G. Dobos, Gy. Vida, Z. Tóth, K. Josepovits. Microsc. Microanal. 11, 567 (2005).

22. Á. Böröczki, G. Dobos, K. Josepovits, Gy. Hárs. Appl. Surf. Sci. 252, 8309 (2006).

23. Niimi. U.S. 2004/0119392 A1 patent application. 